\title{
GLOBAL EXISTENCE FOR A 2D INCOMPRESSIBLE VISCOELASTIC MODEL WITH SMALL STRAIN*
}

\author{
ZHEN LEI ${ }^{\dagger}$, CHUN LIU $\ddagger$, AND YI ZHOU $\$$
}

\begin{abstract}
In this paper, we continue our previous study towards understanding the twodimensional hydrodynamic systems describing Oldroyd type incompressible viscoelastic fluids. We will decompose the deformation tensor into the strain and rotation components and look at their distinct contributions and structures in the small strain (with respect to viscosity) dynamics. In particular, we prove that there exist classical solutions globally in time if the strain component of the initial deformation is small enough, while we require no assumptions on smallness of the magnitude of the rotation component.
\end{abstract}

Key words. viscoelasticity, partial dissipation system, incompressibility, small strain

AMS subject classifications. 76D03, 76D09, 35M10

\section{Introduction}

Viscoelastic materials include a wide range of fluids with elastic properties, as well as solids with fluid properties. The elastic behavior demonstrated by these materials is attributed to the underlying microstructure or configurations of these materials. The deformation of these structures requires exchange of kinetic energy and various internal elastic energies. This energy exchange is realized through special coupling of the transport of the internal elastic variables and the induced elastic stress. Hence the interaction between these microscopic elastic properties and macroscopic fluid motions can be viewed as a competition between the elastic energy and the kinetic energy, which in many cases can be demonstrated through the fact that the momentum equations can be derived from the Least Action Principle [15, 27, 29, 32, 34].

We start with the standard description of general mechanical evolutions. Any deformation can be represented by a flow map (particle trajectory) $x(t, X), 0 \leq t<T$, a time-dependent family of orientation-preserving diffeomorphisms. $X$ is the original labeling (Lagrangian coordinate) of the particles. $X$ is also referred to as the material/reference coordinate. $x$ is then the observer's (Eulerian) coordinate. In general, the velocity field $u(t, x)$ is defined as the time derivative of the flow map, which is a vector field defined on the Eulerian coordinate:

$$
u(t, x)=x_{t}(t, X(t, x)) .
$$

For Newtonian fluids $[13,42]$ and those non-Newtonian fluids with stress tensor (nonlinearly) determined by the strain rate only, such as the viscoplastic fluids proposed in $[5,14]$, the dilatant fluids [12, 43] and those studied by [20, 26, 27, 35], there

*Received: May 1, 2007; accepted: May 9, 2007. Communicated by Weinan E.

Z. Lei was partially supported by LGH under grant 1140003 . Y. Zhou was partially supported by the National Science Foundation of China under grant 10225102, a 973 project of the National Sciential Foundation of China and a Doctoral Program Foundation of the Ministry of Education of China. C. Liu was partially supported by National Science Foundation under grants NSF-DMS 0405850 and NSF-DMS 0509094.

${ }^{\dagger}$ School of Mathematics and Statistics, Northeast Normal University, Changchun 130024, P. R. China; School of Mathematical Sciences, Fudan University, Shanghai 200433, P. R. China (leizhn@yahoo.com).http://www.acm.caltech.edu/ zhenlei/

${ }_{\ddagger}^{\ddagger}$ Department of Mathematics, Pennsylvania State University, State college, PA 16802, (liu@math.psu.edu). http://www.math.psu.edu/ liu/

$\$$ School of Mathematics, Fudan University, Shanghai 200433, P. R. China; Key Laboratory of Mathematics for Nonlinear Sciences (Fudan University), Ministry of Education, P. R. China (yizhou@fudan.ac.cn). http://homepage.fudan.edu.cn/ yizhou/index.htm 
is no "elastic" energy, due to the fact that the systems governing the dynamics do not involve the deformation tensor:

$$
F(t, x)=H(t, X(t, x)),
$$

where $H(t, X)=\frac{\partial x}{\partial X}(t, X)$. On the other hand, the description of any evolution of a pattern/configuration, including those of internal microstructures and morphologies, requires the full information of this deformation tensor. Noticing that the density function only depends on the determinant of $F$. We can distinguish the notion of "elastic" energies by those which involve the full information of $F$ (or $H$ ).

The special memory effects in the non-Newtonian fluids $[6,32,34,37,40]$ can also be attributed to the presence of the deformation tensor.

The chain rule immediately gives the following transport equation of $F(t, x)[15$, $27,29,32,34]$ :

$$
F_{t}+u \cdot \nabla F=\nabla u F
$$

which stands for $\partial_{t} F_{i j}+u \cdot \nabla F_{i j}=\nabla_{k} u_{i} F_{k j}$. This equation can also be regarded as the compatibility condition between the velocity field $u$ and the deformation tensor $F$. We will adopt the notation that the derivatives with respect to the spatial coordinate $x_{k}$ will be denoted by $\nabla_{k}$, for $k=1,2$.

For homogeneous, hyperelastic and isotropic materials, the action functional takes the form

$$
A(x)=\int_{0}^{T} \int_{\Omega_{0}} \frac{1}{2}\left|x_{t}(t, X)\right|^{2}-\widetilde{W}(H) d X d t .
$$

Here the density in the undeformed configuration, $\rho_{0}(X)$, has been chosen to be 1. $\Omega_{0}$ represents the original domain occupied by the material under consideration, and $\Omega_{t}$ will be deformed domain. Note that with zero velocity boundary condition, $\Omega_{0}=\Omega_{t}=\Omega$. $\widetilde{W}(H)$ is the elastic energy functional which can be decomposed into a normal part $h(\rho)$ and a tangential part $W(H)$, namely $\widetilde{W}(H)=W(H)+h(\rho) . h(\rho)$ is determined by the determinant of $H$. W(H) is the elastic strain energy functional.

Formally, the Least Action Principle yields the force balance equations (momentum equations) by taking the first variation of $A(x)$ with respect to the flow map $x$ :

$$
\frac{\delta A}{\delta x}=0
$$

The result is in the weak form as:

$$
\int_{0}^{T} \int_{\Omega_{0}} x_{t}(t, X) \cdot y_{t}(t, X)-\frac{\partial \widetilde{W}(H)}{\partial H_{i j}} \frac{\partial y_{i}}{\partial X_{j}} d X d t=0,
$$

where $y$ is the infinitesimal generator of $x$, which is in the tangent space of that of $x$. In case of incompressible fluids, $x$ is a volume preserving diffeomorphism and $y$ will be divergence free, i.e. $\nabla_{x} \cdot y=0$.

We note the following well-known identities:

$$
\frac{\partial \operatorname{det} H}{\partial H}=H^{-T} \operatorname{det} H, \quad \frac{\partial \rho}{\partial H}=\frac{\partial \frac{1}{\operatorname{det} H}}{\partial H}=-\rho H^{-T},
$$


where $H^{-T}$ denotes the transpose of the inverse matrix of $H$.

Now we can present the following momentum equation in Lagrangian coordinates as:

$$
x_{t t}+\nabla_{X} \cdot\left(H^{-T} p \operatorname{det} H\right)=\nabla_{X} \cdot \frac{\partial W(H)}{\partial H} .
$$

Here we use the notation $(\nabla \cdot H)_{i}=\nabla_{j} H_{i j}$. Moreover, the term $\frac{\partial W(H)}{\partial H}$ on the right hand side of the equation is the Piola-Kirchhoff stress tensor [15]. This is also equivalent to the following representation in Eulerian coordinates:

$$
\rho\left(u_{t}+u \cdot \nabla u\right)+\nabla p=\nabla \cdot\left(\frac{1}{\operatorname{det} F} \frac{\partial W(F)}{\partial F} F^{T}\right),
$$

where $p$ is the hydrostatic pressure, satisfying $p(\rho)=\rho^{2} h^{\prime}(\rho)$ (In more conventional notation, with internal energy being $\int_{\Omega} g(\rho) d x$, the pressure will satisfy $p^{\prime}(\rho)=\rho g^{\prime \prime}(\rho)$.) The term $\frac{1}{\operatorname{det} F} \frac{\partial W(F)}{\partial F} F^{T}$ is the Cauchy-Green tensor (for the corresponding PiolaKirchhoff stress tensor $\frac{\partial W(F)}{\partial F}$ ). This is the Eulerian description of elasticity.

The viscosity term can either be included through the a priori postulation of the dissipative energies as those in $[11,8]$ or through the additional consideration involving the thermo-fluctuation effects [36]. The resulting momentum equation will be:

$$
\rho\left(u_{t}+u \cdot \nabla u\right)+\nabla p=\mu(\Delta u+\nabla \nabla \cdot u)+\nabla \cdot\left(\frac{1}{\operatorname{det} F} \frac{\partial W(F)}{\partial F} F^{T}\right),
$$

and the corresponding incompressible case will be:

$$
u_{t}+u \cdot \nabla u+\nabla p=\mu \Delta u+\nabla \cdot\left(\frac{\partial W(F)}{\partial F} F^{T}\right),
$$

where the pressure $p$ plays the role of the Lagrangian multiplier for the incompressibility constraint $\nabla \cdot u=0$.

If we restrict ourselves to the cases of Hookean elasticity, i.e., $W(F)=\frac{1}{2}|F|^{2}$, the full system can we written as:

$$
\left\{\begin{aligned}
u_{t}+u \cdot \nabla u+\nabla p & =\mu \Delta u+\nabla \cdot\left(F F^{T}\right), \\
F_{t}+u \cdot \nabla F & =\nabla u F \\
\nabla \cdot u & =0 .
\end{aligned}\right.
$$

All the results and the techniques of this paper are valid in the case of general strain energy function $W(F)$. For more details, see Rem. 4.5.

In this paper, we are interested in the global existence of classical solutions for the Cauchy problem or periodic initial-boundary value problem of the system (1.12) in two space dimensions, with small strain conditions. More precisely, the deformation tensor $F$ can be decomposed into a strain part and a rotational part; if we impose the condition that the initial values of the strain part are small with respect to the viscosity (see section 2), and prove that the system has global classical solutions.

The main difficulties for obtaining global existence for system (1.12) with small data (with respect to the viscosity $\mu$ ) lie in the fact that the system is only partially dissipative. In the presence of the dissipative term $\Delta u$ in the momentum equation, we need not impose the null condition on the strain energy function $W(F)$ which is a 
necessary condition when studying elasticity or nonlinear wave equations $[3,4,7,22$, 38]. This is because the system is not scaling-invariant anymore, in contrast to other inviscid problems. This prevents the utilization of the hyperbolic dispersive estimate methods which are based on Lorentz invariance. These methods, often referred to as the generalized energy methods, were originally developed by Klainerman for the construction of solutions to nonlinear wave equations [21] and were later modified by Klainerman and Sideris to treat the case of elasticity with a smaller number of generators. The work in the last part does not rely on the Lorentz invariance but only on the scaling invariance $[24,38,39]$. It is also clear that the usual energy method is not valid here since there is no dissipation from the deformation tensor $F$.

Complex fluids have received a great deal of interest in recent years. In the 2-D incompressible case of viscoelasticity, Lin, Liu and Zhang [32] proved the global existence of classical solutions for near-equilibrium cases. In fact, the method provided a general framework to deal with systems with partial dissipation. Lei and Zhou [29] showed that global solutions of (1.12) can be obtained as the limit of solutions of the compressible viscoelastic system (1.10) as the Mach number tends to 0 . The results has been generalized to the 3-D case by Lei, Liu and Zhou [30].

The corresponding results for the 2-D inviscid cases are still open, with the most up-to-date results being those in [3, 4]. In three-space-dimensional, compressible cases, both Agemi [1] and Sideris [38] proved global existence of small-data classical solutions for elastic waves under the same kind of null conditions. The former's proof relies on direct estimation of the fundamental solution, while the latter's proof utilized an additional set of weighted $L^{2}$ estimates. Recently, the corresponding results in the incompressible case were given by Sideris and Thomases [39] via the incompressible limit. However, their methods cannot be applied directly to the viscoelastic case since scaling invariance of systems plays an important role in their proof.

The linear transport equation for deformation tensor $F$ cannot be treated directly in the framework of [25]. Nonetheless, one may apply the div-curl lemma [41] to obtain a weak solution [34] in some special cases. In general, it is more difficult to obtain the compactness for the Cauchy-Green tensor of sequences of solutions, see [32, 33, 34].

K. O. Friedrich observed that the smallness of the strain in nonlinear elasticity can be realized through the polar decomposition of the deformation tensor. The corresponding nonlinear elasticity problem was studied in the famous work of F. John [19]. Liu and Walkington [34] considered approximating systems resulting from the special linearization of the original system with respect to the strain. Our decomposition here is motivated by those works, but with distinct advantages in the analysis procedure. This paper provides the first such result in the dynamical cases.

In section 2, we convert the original system to the small strain case by decomposing the deformation tensor $F$ into its rotation and stretching parts. In section 3, we study the local existence of solutions of the system (1.12). In section 4 , we prove the global existence of classical solutions of the system.

\section{Rotation-strain decomposition}

We start with the transport equation for the deformation tensor $F$. First, we decompose $F$ into its strain part $V$ and rotation part $Q$.

$$
F=(I+V) Q .
$$

We point out that here we relax the usual symmetric constraint on $V$. Instead, we require that $Q$ satisfies the equation

$$
Q_{t}+u \cdot \nabla Q=\omega(u) Q
$$


where

$$
\omega(u)=\frac{\nabla u-\nabla^{T} u}{2}
$$

is the skew-symmetric part of the strain rate tensor, i.e., the vorticity tensor. Hence $Q$ is a rotation matrix. In particular,

$$
Q Q^{T}=I
$$

for all time if the initial data satisfies $Q_{0} Q_{0}^{T}=I$. Moreover, we can write this $2 \times 2$ orthogonal matrix in the form:

$$
Q=\left(\begin{array}{cc}
\cos \theta & -\sin \theta \\
\sin \theta & \cos \theta
\end{array}\right)
$$

The equation (2.2) can then be reformulated as:

$$
\theta_{t}+u \cdot \nabla \theta=\frac{\nabla_{1} u_{2}-\nabla_{2} u_{1}}{2} .
$$

This gives the following important equation:

$$
\partial_{t} \nabla^{\perp} \theta+u \cdot \nabla \nabla^{\perp} \theta+\left(\begin{array}{c}
-\nabla_{2} u \cdot \nabla \theta \\
\nabla_{1} u \cdot \nabla \theta
\end{array}\right)=\frac{1}{2} \Delta u .
$$

Here we use the notation that

$$
\nabla^{\perp} \theta=\left(\begin{array}{c}
-\nabla_{2} \theta \\
\nabla_{1} \theta
\end{array}\right)
$$

Inserting (2.1), (2.2) and (2.3) into the second equation of system (1.12) (the transport equation for $F$ ), one obtains:

$$
\left((I+V)_{t}+u \cdot \nabla(I+V)\right) Q+(I+V)\left(Q_{t}+u \cdot \nabla Q\right)=\nabla u(I+V) Q .
$$

This equation yields the evolution of the strain component $V$ :

$$
V_{t}+u \cdot \nabla V=D(u)+\nabla u V-V \omega(u),
$$

where

$$
D(u)=\frac{\nabla u+\nabla^{T} u}{2}
$$

is the symmetric part of the strain rate tensor.

In summary, the final system becomes

$$
\left\{\begin{aligned}
\nabla \cdot u & =0, \\
u_{t}+u \cdot \nabla u+\nabla p & =\mu \Delta u+\nabla \cdot\left(V V^{T}\right)+\nabla \cdot V+\nabla \cdot V^{T}, \\
V_{t}+u \cdot \nabla V & =D(u)+\nabla u V-V \omega(u), \\
\partial_{t} \nabla^{\perp} \theta+u \cdot \nabla \nabla^{\perp} \theta+\left(\begin{array}{c}
-\nabla_{2} u \cdot \nabla \theta \\
\nabla_{1} u \cdot \nabla \theta
\end{array}\right) & =\frac{1}{2} \Delta u .
\end{aligned}\right.
$$


We remark that the first three equation on $(u, V)$ constitute a closed system, without involving the variable $\theta$. However, as we will see in the following sections, the physical constraint of the original viscoelastic problem will involve this "phantom" variable $\theta$, through the incompressibility conditions.

We are interested in the Cauchy problem or the periodic initial-boundary value problem of the above system (2.10). The domain $\Omega$ under consideration can be a 2-dimension torus or the entire space $R^{2}$. The initial data is of the form:

$$
u(0, x)=u_{0}(x), \quad V(0, x)=V_{0}(x), \quad \nabla \theta(0, x)=\nabla \theta_{0}(x) .
$$

From now on, we will focus on the above system (2.10). Moreover, we will impose the following additional constraints on the initial data:

$$
\left\{\begin{aligned}
\nabla \cdot u_{0} & =0, \\
\operatorname{det}\left(I+V_{0}\right) & =1, \\
\nabla \cdot V_{0}^{T} & =\nabla^{\perp} \theta_{0}+\left(\begin{array}{c}
-V_{012} \nabla_{1} \theta_{0}-V_{022} \nabla_{2} \theta_{0} \\
V_{011} \nabla_{1} \theta_{0}+V_{021} \nabla_{2} \theta_{0}
\end{array}\right) .
\end{aligned}\right.
$$

These constraints are derived from physical consideration of the original viscoelastic system. As explained at the end of this section, all three are consequences of the incompressibility. In particular, the third equation is derived from the special constraint $\nabla \cdot F^{T}=0($ see $[30,32])$.

The following two lemmas demonstrate some special structures under the new system, in particular, under the constraints on the initial data.

LEMMA 2.1. Assume that the second equality of (2.12) is satisfied and $(u, V, \theta)$ is the solution of system (2.10). Then we have

$$
\operatorname{det}(I+V)=1
$$

for all later times $t \geq 0$.

Proof. Applying the identity (1.7), together with the first and third equations of (2.10), we have

$$
\begin{aligned}
& (\operatorname{det}(I+V))_{t}+u \cdot \nabla(\operatorname{det}(I+V)) \\
= & \operatorname{det}(I+V)(I+V)_{j i}^{-1}\left[\frac{\nabla_{i} u_{j}+\nabla_{j} u_{i}}{2}+\nabla_{k} u^{i} V_{k j}-V_{i k} \omega_{k j}\right] \\
= & \operatorname{det}(I+V)(I+V)_{j i}^{-1}\left[\nabla_{k} u^{i}\left(\delta_{k j}+V_{k j}\right)-\left(\delta_{i k}+V_{i k}\right) \omega_{k j}\right] \\
= & \operatorname{det}(I+V) \nabla \cdot u=0 .
\end{aligned}
$$

Hence the result of the lemma follows.

From the next lemma, we can see that the unknown variable $\theta$, i.e. $Q$, is coupled in the system through some special structure, despite the fact that the equations of $(u, V)$ are already closed.

Lemma 2.2. Assume that the initial data satisfies the constraints $(2.12)$, and $(u, V, \theta)$ solves the system (2.10), Then for all the time $t \geq 0$, we have

$$
\nabla \cdot V^{T}=\nabla^{\perp} \theta+\left(\begin{array}{c}
-V_{12} \nabla_{1} \theta-V_{22} \nabla_{2} \theta \\
V_{11} \nabla_{1} \theta+V_{21} \nabla_{2} \theta
\end{array}\right) .
$$


Proof. First of all, by transposing the third equation of (2.10) and applying $\nabla$. to the resulting equation, we have

$$
\partial_{t}\left(\nabla \cdot V^{T}\right)+u \cdot \nabla\left(\nabla \cdot V^{T}\right)+\nabla_{k} u \cdot \nabla V^{T}=\frac{1}{2} \Delta u+\nabla \cdot\left(V^{T} \nabla u^{T}+\omega(u) V^{T}\right) .
$$

Combing this with the fourth equation of (2.10), we have

$$
\begin{aligned}
& \partial_{t}\left[\nabla \cdot V^{T}-\left(\begin{array}{c}
-\nabla_{2} \theta \\
\nabla_{1} \theta
\end{array}\right)\right]+u \cdot \nabla\left[\nabla \cdot V^{T}-\left(\begin{array}{c}
-\nabla_{2} \theta \\
\nabla_{1} \theta
\end{array}\right)\right] \\
& =\left(\begin{array}{c}
-\nabla_{2} u \cdot \nabla \theta \\
\nabla_{1} u \cdot \nabla \theta
\end{array}\right)-\nabla_{k} u \cdot \nabla V^{T}+\nabla \cdot\left(V^{T} \nabla u^{T}+\omega(u) V^{T}\right) .
\end{aligned}
$$

On the other hand, by the last two equations of (2.10), we have

$$
\begin{aligned}
& \partial_{t}\left(\begin{array}{c}
V_{12} \nabla_{1} \theta+V_{22} \nabla_{2} \theta \\
-V_{11} \nabla_{1} \theta-V_{21} \nabla_{2} \theta
\end{array}\right)+u \cdot \nabla\left(\begin{array}{c}
V_{12} \nabla_{1} \theta+V_{22} \nabla_{2} \theta \\
-V_{11} \nabla_{1} \theta-V_{21} \nabla_{2} \theta
\end{array}\right) \\
&=\left(\begin{array}{c}
-V_{12} \nabla_{1} u \cdot \nabla \theta+\frac{1}{2} V_{12} \Delta u_{2}+\nabla_{1} \theta \frac{\nabla_{1} u_{2}+\nabla_{2} u_{1}}{2}+\nabla_{1} \theta \nabla_{k} u_{1} V_{k 2}-\nabla_{1} \theta V_{1 k} \omega_{k 2} \\
V_{11} \nabla_{1} u \cdot \nabla \theta-\frac{1}{2} V_{11} \Delta u_{2}-\nabla_{1} \theta \nabla_{1} u_{1}-\nabla_{1} \theta \nabla_{k} u_{1} V_{k 1}+\nabla_{1} \theta V_{1 k} \omega_{k 1}
\end{array}\right) \\
& \quad+\left(\begin{array}{c}
-V_{22} \nabla_{2} u \cdot \nabla \theta-\frac{1}{2} V_{22} \Delta u_{1}+\nabla_{2} \theta \nabla_{2} u_{2}+\nabla_{2} \theta \nabla_{k} u_{2} V_{k 2}-\nabla_{2} \theta V_{2 k} \omega_{k 2} \\
V_{21} \nabla_{2} u \cdot \nabla \theta+\frac{1}{2} V_{21} \Delta u_{1}-\nabla_{2} \theta \frac{\nabla_{1} u_{2}+\nabla_{2} u_{1}}{2}-\nabla_{2} \theta \nabla_{k} u_{2} V_{k 1}+\nabla_{2} \theta V_{2 k} \omega_{k 1}
\end{array}\right) .
\end{aligned}
$$

Adding these two equations together and noting that $\nabla \cdot u=0$ and $\omega(u)_{11}=$ $\omega(u)_{22}=0$, we have

$$
\begin{aligned}
& \partial_{t}\left[\nabla \cdot V^{T}-\left(\begin{array}{c}
-\nabla_{2} \theta \\
\nabla_{1} \theta
\end{array}\right)+\left(\begin{array}{c}
V_{12} \nabla_{1} \theta+V_{22} \nabla_{2} \theta \\
-V_{11} \nabla_{1} \theta-V_{21} \nabla_{2} \theta
\end{array}\right)\right] \\
& +u \cdot \nabla\left[\nabla \cdot V^{T}-\left(\begin{array}{c}
-\nabla_{2} \theta \\
\nabla_{1} \theta
\end{array}\right)+\left(\begin{array}{c}
V_{12} \nabla_{1} \theta+V_{22} \nabla_{2} \theta \\
-V_{11} \nabla_{1} \theta-V_{21} \nabla_{2} \theta
\end{array}\right)\right] \\
& =\left(\begin{array}{c}
-V_{12} \nabla_{1} u \cdot \nabla \theta+\frac{1}{2} V_{12} \Delta u_{2}+\nabla_{1} \theta \frac{\nabla_{1} u_{2}+\nabla_{2} u_{1}}{2}+\nabla_{1} \theta \nabla_{k} u_{1} V_{k 2}-\nabla_{1} \theta V_{1 k} \omega_{k 2} \\
V_{11} \nabla_{1} u \cdot \nabla \theta-\frac{1}{2} V_{11} \Delta u_{2}-\nabla_{1} \theta \nabla_{1} u_{1}-\nabla_{1} \theta \nabla_{k} u_{1} V_{k 1}+\nabla_{1} \theta V_{1 k} \omega_{k 1}
\end{array}\right) \\
& +\left(\begin{array}{c}
-V_{22} \nabla_{2} u \cdot \nabla \theta-\frac{1}{2} V_{22} \Delta u_{1}+\nabla_{2} \theta \nabla_{2} u_{2}+\nabla_{2} \theta \nabla_{k} u_{2} V_{k 2}-\nabla_{2} \theta V_{2 k} \omega_{k 2} \\
V_{21} \nabla_{2} u \cdot \nabla \theta+\frac{1}{2} V_{21} \Delta u_{1}-\nabla_{2} \theta \frac{\nabla_{1} u_{2}+\nabla_{2} u_{1}}{2}-\nabla_{2} \theta \nabla_{k} u_{2} V_{k 1}+\nabla_{2} \theta V_{2 k} \omega_{k 1}
\end{array}\right) \\
& +\left(\begin{array}{c}
-\nabla_{2} u \cdot \nabla \theta \\
\nabla_{1} u \cdot \nabla \theta
\end{array}\right)-\nabla_{k} u \cdot \nabla V^{T}+\nabla \cdot\left(V^{T} \nabla u^{T}+\omega(u) V^{T}\right) \\
& =\left(\begin{array}{c}
\nabla_{1} \theta \frac{\nabla_{1} u_{2}+\nabla_{2} u_{1}}{2}+\nabla_{2} \theta \nabla_{2} u_{2} \\
-\nabla_{1} \theta \nabla_{1} u_{1}-\nabla_{2} \theta \frac{\nabla_{1} u_{2}+\nabla_{2} u_{1}}{2}
\end{array}\right)+\left(\begin{array}{c}
-\nabla_{2} u \cdot \nabla \theta \\
\nabla_{1} u \cdot \nabla \theta
\end{array}\right) \\
& +\left(\begin{array}{l}
+\frac{1}{2} V_{12} \Delta u_{2}-\frac{1}{2} V_{22} \Delta u_{1} \\
-\frac{1}{2} V_{11} \Delta u_{2}+\frac{1}{2} V_{21} \Delta u_{1}
\end{array}\right)-\nabla_{k} u \cdot \nabla V^{T}+\nabla \cdot\left(V^{T} \nabla u^{T}+\omega(u) V^{T}\right) \\
& +\left(\begin{array}{c}
-V_{12} \nabla_{1} u \cdot \nabla \theta+\nabla_{1} \theta \nabla_{k} u_{1} V_{k 2}-\nabla_{1} \theta V_{1 k} \omega_{k 2} \\
V_{11} \nabla_{1} u \cdot \nabla \theta-\nabla_{1} \theta \nabla_{k} u_{1} V_{k 1}+\nabla_{1} \theta V_{1 k} \omega_{k 1}
\end{array}\right) \\
& +\left(\begin{array}{c}
-V_{22} \nabla_{2} u \cdot \nabla \theta+\nabla_{2} \theta \nabla_{k} u_{2} V_{k 2}-\nabla_{2} \theta V_{2 k} \omega_{k 2} \\
V_{21} \nabla_{2} u \cdot \nabla \theta-\nabla_{2} \theta \nabla_{k} u_{2} V_{k 1}+\nabla_{2} \theta V_{2 k} \omega_{k 1}
\end{array}\right) \\
& =-\omega(u) \nabla^{\perp} \theta+\omega(u) \nabla \cdot V^{T} \\
& +\left(\begin{array}{c}
-V_{12} \nabla_{1} u \cdot \nabla \theta+\nabla_{1} \theta \nabla_{1} u_{1} V_{12}+\nabla_{1} \theta \nabla_{2} u_{1} V_{22}-\nabla_{1} \theta V_{11} \omega_{12} \\
V_{11} \nabla_{1} u \cdot \nabla \theta-\nabla_{1} \theta \nabla_{1} u_{1} V_{11}-\nabla_{1} \theta \nabla_{2} u_{1} V_{21}+\nabla_{1} \theta V_{12} \omega_{21}
\end{array}\right)
\end{aligned}
$$




$$
\begin{aligned}
& +\left(\begin{array}{c}
-V_{22} \nabla_{2} u \cdot \nabla \theta+\nabla_{2} \theta \nabla_{1} u_{2} V_{12}+\nabla_{2} \theta \nabla_{2} u_{2} V_{22}-\nabla_{2} \theta V_{21} \omega_{12} \\
V_{21} \nabla_{2} u \cdot \nabla \theta-\nabla_{2} \theta \nabla_{1} u_{2} V_{11}-\nabla_{2} \theta \nabla_{2} u_{2} V_{21}+\nabla_{2} \theta V_{22} \omega_{21}
\end{array}\right) \\
& =-\omega(u) \nabla^{\perp} \theta+\omega(u) \nabla \cdot V^{T}+\left(\begin{array}{c}
-\nabla_{1} \theta V_{11} \omega_{12}-\nabla_{2} \theta V_{21} \omega_{12} \\
\nabla_{1} \theta V_{12} \omega_{21}+\nabla_{2} \theta V_{22} \omega_{21}
\end{array}\right) \\
& =\omega(u)\left[\nabla \cdot V^{T}-\left(\begin{array}{c}
-\nabla_{2} \theta \\
\nabla_{1} \theta
\end{array}\right)+\left(\begin{array}{c}
V_{12} \nabla_{1} \theta+V_{22} \nabla_{2} \theta \\
-V_{11} \nabla_{1} \theta-V_{21} \nabla_{2} \theta
\end{array}\right)\right] .
\end{aligned}
$$

This implies the result of the lemma if we choose the corresponding initial conditions.

From these lemmas, we can see that the constraints (2.12) on the initial data are consistent with physical incompressibility, rather than merely mathematical technicality. In particular, all three conditions are consequences of incompressibility. The two lemmas above represent the special structures of the system induced by the incompressibility.

To elaborate, we look back at the macroscopic incompressibility condition:

$$
\operatorname{det} F=1 .
$$

The second assumption of (2.12) is the direct consequence of this, since $Q$ is an orthogonal matrix. Next, recalling the conservation law for mass $\rho \operatorname{det} F=1$ and its corresponding transport form,

$$
\rho_{t}+\nabla \cdot(\rho u)=0
$$

we recover the incompressible constraint on $u$,

$$
\nabla \cdot u=0 .
$$

We can view the third assumption of (2.12), hence (2.14) from another angle. Recall the following proposition for any incompressible mechanical deformations, which was first derived in [34] and later extensively used in [30, 32].

Proposition 2.3. If we set the density in the reference configuration equal to one, then we have

$$
(\nabla \cdot F)_{t}+u \cdot(\nabla \cdot F)=0 .
$$

Moreover, under suitable initial conditions, $\nabla_{j} F_{j i}=0$ for $i=1,2$.

In other words, we have

$$
\begin{aligned}
0 & =Q_{l j}\left(\nabla_{i} Q_{i j}+Q_{k j} \nabla_{i} V_{i k}+V_{i k} \nabla_{i} Q_{k j}\right) \\
& =\nabla_{i} V_{i l}+Q_{l j} \nabla_{i} Q_{i j}+Q_{l j} V_{i k} \nabla_{i} Q_{k j},
\end{aligned}
$$

which is equivalent to the equation:

$$
\left(\begin{array}{c}
\nabla_{i} V_{i 1} \\
\nabla_{j} V_{j 2}
\end{array}\right)=\left(\begin{array}{c}
-\nabla_{2} \theta \\
\nabla_{1} \theta
\end{array}\right)+\left(\begin{array}{c}
-V_{12} \nabla_{1} \theta-V_{22} \nabla_{2} \theta \\
V_{11} \nabla_{1} \theta+V_{21} \nabla_{2} \theta
\end{array}\right)
$$

For convenience, we can write the constraint (2.14) on $V$ in the expression:

$$
\nabla \cdot V^{T}=\nabla^{\perp} \theta+J V^{T} \nabla \theta
$$

where

$$
J=\left(\begin{array}{cc}
0 & -1 \\
1 & 0
\end{array}\right)
$$




\section{Local existence of classical solutions for initial value problems}

The proof of the local existence of the classical solutions is standard once we note that the first three equations of system (2.10) constitute a closed system. One can obtain the local classical solution of the closed system by the usual Galerkin's method, see $[32,34,42]$ for a detailed explanation. Alternatively, one can also obtain the local classical solution via the method of the incompressible limit as in [28].

Imitating the argument in [32], we can obtain the following local existence results.

Lemma 3.1. Let $k \geq 2$ be a positive integer, $u_{0} \in H^{k}(\Omega), V_{0} \in H^{k}(\Omega)$, and assumption (2.12) be satisfied. Then there exists a positive time $T$, which depends only on $\left\|u_{0}\right\|_{H^{2}(\Omega)}$ and $\left\|V_{0}\right\|_{H^{2}(\Omega)}$, such that the Cauchy problem or the periodic initialboundary value problem for the system consisting of the first three equations of (2.10) with initial data (2.11) possesses a unique solution in the time interval $[0, T]$ with

$$
\left\{\begin{array}{l}
\partial_{t}^{j} \nabla^{\alpha} u \in L^{\infty}\left(0, T ; H^{k-2 j-|\alpha|}(\Omega)\right) \cap L^{2}\left(0, T ; H^{k-2 j-|\alpha|+1}(\Omega)\right), \\
\partial_{t}^{j} \nabla^{\alpha} V \in L^{\infty}\left(0, T ; H^{k-2 j-|\alpha|}(\Omega)\right)
\end{array}\right.
$$

for all $j, \alpha$ satisfying $2 j+|\alpha| \leq k$. Moreover, if $T^{*}$ is the maximal time of existence, then

$$
\int_{0}^{T^{*}}\|u\|_{H^{2}(\Omega)}^{2} d t=+\infty
$$

This will in turn gives the corresponding results for the last equation of (2.10):

Lemma 3.2. Under the assumptions of Lemma 3.1, we further assume that

$$
\partial_{t}^{j} \nabla^{\alpha} u \in L^{\infty}\left(0, T ; H^{k-2 j-|\alpha|}(\Omega)\right) \cap L^{2}\left(0, T ; H^{k-2 j-|\alpha|+1}(\Omega)\right),
$$

for some positive constant $T$ and all $j, \alpha$ satisfying $2 j+|\alpha| \leq k$. Then $\nabla \theta$ can be uniquely determined by the last equation of (2.10) with initial data $\nabla \theta_{0}$ and

$$
\partial_{t}^{j} \nabla^{\alpha} \nabla \theta \in L^{\infty}\left(0, T ; H^{k-2 j-|\alpha|-1}(\Omega)\right),
$$

for all $j, \alpha$ satisfying $2 j+|\alpha| \leq k$, provided $\nabla \theta_{0} \in H^{k-1}(\Omega)$.

In summary, we can state the tesult for the local existence of classical solutions for the system (2.10)-(2.11).

TheOREM 3.3. Let $k \geq 2$ be a positive integer, $u_{0} \in H^{k}(\Omega), V_{0} \in H^{k}(\Omega)$ and $\nabla \theta_{0} \in$ $H^{k-1}(\Omega)$. Then there exists a positive time $T$, which depends only on $\left\|u_{0}\right\|_{H^{2}(\Omega)}$, $\left\|V_{0}\right\|_{H^{2}(\Omega)}$ and $\left\|\nabla \theta_{0}\right\|_{H^{1}(\Omega)}$, such that the Cauchy initial value problems or the periodic initial-boundary value problem for the system (2.10)-(2.11) possesses a unique solution in the time interval $[0, T]$ with

$$
\left\{\begin{array}{l}
\partial_{t}^{j} \nabla^{\alpha} u \in L^{\infty}\left(0, T ; H^{k-2 j-|\alpha|}(\Omega)\right) \cap L^{2}\left(0, T ; H^{k-2 j-|\alpha|+1}(\Omega)\right) \\
\partial_{t}^{j} \nabla^{\alpha} V \in L^{\infty}\left(0, T ; H^{k-2 j-|\alpha|}(\Omega)\right)
\end{array}\right.
$$

for all $j, \alpha$ satisfying $2 j+|\alpha| \leq k$ and

$$
\partial_{t}^{j} \nabla^{\alpha} \nabla \theta \in L^{\infty}\left(0, T ; H^{k-2 j-|\alpha|-1}(\Omega)\right)
$$


for for all $j, \alpha$ satisfying $2 j+|\alpha| \leq k-1$. Moreover, if $T^{*}$ is the maximal time of existence, then

$$
\int_{0}^{T^{*}}\|u\|_{H^{2}(\Omega)}^{2} d t=+\infty
$$

\section{Global existence for small-strain initial conditions}

In this section, we will prove the global existence of classical solutions for the system (2.10)-(2.11), with a small initial condition satisfying the assumptions (2.12). To avoid the complications due to the boundary conditions, we concentrate below on the situation concerning only the entire space. In fact, the periodic and smooth bounded domain cases can also be treated by more lengthy argument. However the procedure only involves a more technical complications compared to the proofs below.

As in the cases that were studied in $[30,32,34]$, the main difficulty lies in the partial dissipation, as well as the loss of scaling invariance of the system. The presence of a damping mechanism on $u$ becomes an obstacle when one wants to utilize the techniques involving the combination of Klainerman's inequality with weighted $L^{2}$ estimates, even in three space dimensional cases $[21,22,38,39]$.

The main achievement of the paper is to recognize the special structures of the system which are specifically due to the incompressible mechanical deformation. Such structures were revealed by Lemma 2.2. The specific manipulation of these structures enables us to obtain certain dissipative estimates for the deformation tensor $F$, which in turn yields the unusual higher-order energy methods to get a priori estimates of solutions. A similar idea has been employed in [32], and later in $[29,30]$. In this paper, we refine our near-equilibrium assumptions in $[32,30]$ to more precise physical considerations, that is, the smallness on the strain (not on the rotation) part of the deformation tensor. Such consideration was used in [34] to attempt to derive a modified system which they can prove the existence of global weak solution. Here we study the exact transformation of the original system. Consideration of such smallness in strain from nonlinear elasticity was also employed in the earlier work of F. John [18].

From now on, we will denote by $\|\cdot\|$ the $L^{2}\left(R^{2}\right)$ norm and $\langle\cdot\rangle$, the inner product of standard Euclidean space $R^{1}, R^{2}$, or $R^{4}$.

We start with the basic energy estimate for the momentum equation. By taking the $L^{2}$ inner product of the middle two equations of (2.10) with $u$ and $V$, respectively, and using integration by parts, one obtains

$$
\begin{aligned}
& \frac{1}{2} \frac{d}{d t} \int_{R^{2}}\left(|u|^{2}+|V|^{2}\right) d x+\mu\|\nabla u\|^{2} \\
= & -\int_{R^{2}} u \cdot \nabla \frac{|u|^{2}+|V|^{2}}{2}+\langle u, \nabla p\rangle d x+\int_{R^{2}}\langle V, \nabla u V\rangle d x \\
& +\int_{R^{2}}\left\langle u, \nabla \cdot V+\nabla \cdot V^{T}\right\rangle+\langle V, D(u)\rangle d x \\
& +\int_{R^{2}}\left\langle u, \nabla \cdot\left(V V^{T}\right)\right\rangle d x-\int_{R^{2}}\langle V, V \omega(u)\rangle d x .
\end{aligned}
$$


On the other hand, we have

$$
\begin{aligned}
\frac{1}{2} \frac{d}{d t} \int_{R^{2}} 2 \operatorname{tr} V d x= & -\int_{R^{2}} u \cdot \nabla \operatorname{tr} V d x+\int_{R^{2}} \operatorname{tr} D(u) d x \\
& +\int_{R^{2}} \operatorname{tr}(\nabla u V-V \omega(u)) d x
\end{aligned}
$$

By (2.3) and (2.9), it is easy to see that:

$$
\begin{gathered}
\int_{R^{2}}\langle V, \nabla u V\rangle+\left\langle u, \nabla \cdot\left(V V^{T}\right)\right\rangle d x=0, \\
\int_{R^{2}}\langle V, V \omega(u)\rangle d x=0, \\
\left.\int_{R^{2}}\langle D(u), V\rangle+\left\langle u, \nabla \cdot V+\nabla \cdot V^{T}\right)\right\rangle d x \\
+\int_{R^{2}} \operatorname{tr}(\nabla u V-V \omega(u)) d x=0 .
\end{gathered}
$$

Thus, we have the basic energy law:

$$
\frac{1}{2} \frac{d}{d t} \int_{R^{2}}\left(|u|^{2}+|V|^{2}+2 \operatorname{tr} V\right) d x+\mu\|\nabla u\|^{2}=0 .
$$

Applying $\Delta$ to the middle two equations of (2.10) and then taking the $L^{2}$ inner product of the resulting equations with $\Delta u$ and $2 \Delta V$, respectively, we obtain

$$
\begin{aligned}
& \frac{1}{2} \frac{d}{d t} \int_{R^{2}}\left(|\Delta u|^{2}+2|\Delta V|^{2}\right) d x+\mu\|\nabla \Delta u\|^{2} \\
&=\int_{R^{2}}\left\langle\Delta \nabla \cdot V+\Delta \nabla \cdot V^{T}, \Delta u\right\rangle+\langle 2 \Delta D(u), \Delta V\rangle d x \quad-\int_{R^{2}}\langle\Delta(u \cdot \nabla u), \Delta u\rangle+2\langle\Delta(u \cdot \nabla V), \Delta V\rangle d x \\
& \quad+\int_{R^{2}}\left\langle\Delta \nabla \cdot\left(V V^{T}\right), \Delta u\right\rangle+2\langle\Delta(\nabla u V), \Delta V\rangle d x \\
& \quad-\int_{R^{2}}\langle\nabla \Delta p, \Delta u\rangle d x-\int_{R^{2}} 2\langle\Delta(V \omega(u)), \Delta V\rangle d x .
\end{aligned}
$$

By integration by parts, one can easily get

$$
\begin{aligned}
\int_{R^{2}}\langle\Delta \nabla \cdot V & \left.+\Delta \nabla \cdot V^{T}, \Delta u\right\rangle+\langle 2 \Delta D(u), \Delta V\rangle d x \\
& -\int_{R^{2}}\langle\nabla \Delta p, \Delta u\rangle=0 .
\end{aligned}
$$

To estimate the rest of the terms of the right side of (4.2), we recall the following well-known Lemma which can be found in the literature $[2,23,31]$. 
Lemma 4.1. Assume $f, g \in H^{s}(\Omega), s \geq 2, \Omega$ being the entire space $R^{2}$ or a bounded domain with smooth boundary $\partial \Omega$ and periodic boundary conditions. Then there exists a universal constant $C$ depending only on $\Omega$ such that

$$
\left\{\begin{array}{l}
\|\Delta(f g)\| \leq C\left(\|f\|_{\infty}\|\Delta g\|+\|g\|_{\infty}\|\Delta f\|\right), \\
\|f\|_{L^{4}} \leq C\|f\|^{\frac{1}{2}}\|\nabla f\|^{\frac{1}{2}} \\
\|\nabla f\|_{L^{4}} \leq C\|\Delta f\|^{\frac{1}{2}}\|f\|_{L^{\infty}}^{\frac{1}{2}} .
\end{array}\right.
$$

Using the last two interpolating inequalities in (4.4), we can estimate the second line of the right side of (4.2) as follows:

$$
\begin{aligned}
& \quad\left|-\int_{R^{2}}\langle\Delta(u \cdot \nabla u), \Delta u\rangle+2\langle\Delta(u \cdot \nabla V), \Delta V\rangle d x\right| \\
& =\mid \int_{R^{2}}\langle\Delta(u \cdot \nabla u)-u \cdot \nabla \Delta u, \Delta u\rangle \\
& \quad+2\langle\Delta(u \cdot \nabla V)-u \cdot \nabla \Delta V, \Delta V\rangle d x \mid \\
& \leq \quad C\left(\|\Delta u\|\|\nabla u\|_{L^{4}}\|\Delta u\|_{L^{4}}+\|\Delta V\|\|\nabla u\|_{\infty}\|\Delta V\|\right. \\
& \left.\quad+\|\Delta V\|\|\nabla V\|_{L^{4}}\|\Delta u\|_{L^{4}}\right) \\
& \leq C(1+\mu)\left(\|u\|_{H^{2}}+\|V\|_{H^{2}}\right)\left(\frac{1}{\mu^{2}}\|\Delta V\|^{2}+\|\nabla u\|^{2}+\|\nabla \Delta u\|^{2}\right) .
\end{aligned}
$$

Here and hereafter, $C$ will denote a generic constant independent of the viscosity $\mu$. The specific meaning of $C$ may vary according to the contex.

Similarly, we can estimate the remaining terms of the right side of (4.2) as follows:

$$
\begin{aligned}
& \left|\int_{R^{2}}\left\langle\Delta \nabla \cdot\left(V V^{T}\right), \Delta u\right\rangle+2\langle\Delta(\nabla u V), \Delta V\rangle d x\right| \\
= & \left|\int_{R^{2}}-\left\langle\Delta\left(V V^{T}\right), \nabla \Delta u\right\rangle+2\langle\Delta(\nabla u V), \Delta V\rangle d x\right| \\
\leq & C\left(\|\nabla \Delta u\|\|\Delta V\|\|V\|_{\infty}+\|\nabla u\|_{\infty}\|\Delta V\|^{2}\right) \\
\leq & C(1+\mu)\left(\|u\|_{H^{2}}+\|V\|_{H^{2}}\right)\left(\frac{1}{\mu^{2}}\|\Delta V\|^{2}+\|\nabla u\|^{2}+\|\nabla \Delta u\|^{2}\right),
\end{aligned}
$$

and

$$
\begin{aligned}
& \left|\int_{R^{2}} 2\langle\Delta(V \omega(u)), \Delta V\rangle d x\right| \\
\leq & C\left(\|\nabla \Delta u\|\|\Delta V\|\|V\|_{\infty}+\|\nabla u\|_{\infty}\|\Delta V\|^{2}\right) \\
\leq & C(1+\mu)\left(\|u\|_{H^{2}}+\|V\|_{H^{2}}\right)\left(\frac{1}{\mu^{2}}\|\Delta V\|^{2}+\|\nabla u\|^{2}+\|\nabla \Delta u\|^{2}\right) .
\end{aligned}
$$

The last steps in (4.6) and (4.7) involve the following estimate:

$$
\begin{aligned}
& \|\nabla u\|_{\infty}\|\Delta V\|^{2} \\
= & \|\Delta V\|\|\Delta V\|\|\nabla u\|_{\infty} \\
\leq & C\|V\|_{H^{2}}\|\Delta V\|(\|\nabla u\|+\|\nabla \Delta u\|) \\
\leq & C(1+\mu)\left(\|u\|_{H^{2}}+\|V\|_{H^{2}}\right)\left(\frac{1}{\mu^{2}}\|\Delta V\|^{2}+\|\nabla u\|^{2}+\|\nabla \Delta u\|^{2}\right) .
\end{aligned}
$$


Finally, combining (4.1)-(4.3) and (4.5)-(4.7), one obtains

$$
\begin{aligned}
& \frac{1}{2} \frac{d}{d t}\left(\|u\|_{H^{2}}^{2}+2\|V\|_{H^{2}}^{2}\right)+\mu\|\nabla u\|_{H^{2}}^{2} \\
\leq & C(1+\mu)\left(\|u\|_{H^{2}}+\|V\|_{H^{2}}\right)\left(\frac{1}{\mu^{2}}\|\Delta V\|^{2}+\|\nabla u\|_{H^{2}}^{2}\right) .
\end{aligned}
$$

Here we observe that the incompressible condition $\operatorname{det} F=1$ gives $\operatorname{tr} V=-\operatorname{det} V$, so $\operatorname{tr} V$ is a higher order term in $V$. This observation will be used again later in the proof.

It is clear from (4.8) that our obstacle now is to find the dissipation of the term $\|\Delta V\|$. We recall that a similar situation occurs in our earlier work [30, 32]. However, the system and the unknown variable are different here.

We introduce the new variable:

$$
w=u+\frac{1}{\mu} \Delta^{-1} \nabla \cdot V
$$

Our goal is to establish dissipative energy estimates for this new variable, more precisely, for $\|\nabla \Delta w\|$, which can help us estimate $\|\Delta V\|$.

By applying $\Delta$ and $\frac{1}{\mu} \nabla$. to the momentum equation and the transport equation of system (2.10) and adding the resulting equations, we have

$$
\begin{aligned}
& \Delta w_{t}+\Delta(u \cdot \nabla u)+\frac{1}{\mu} \nabla \cdot(u \cdot \nabla V)+\nabla \Delta p \\
= & \mu \Delta^{2} w+\Delta \nabla \cdot\left(V V^{T}\right)+\Delta \nabla \cdot V^{T}+\frac{1}{\mu} \nabla \cdot D(u) \\
& +\frac{1}{\mu} \nabla \cdot(\nabla u V-V \omega(u)) .
\end{aligned}
$$

Taking the $L^{2}$ inner product of the above equation (4.10) with $\Delta w$, we obtain that

$$
\begin{aligned}
& \quad \frac{1}{2} \frac{d}{d t}\|\Delta w\|^{2}+\mu\|\nabla \Delta w\|^{2} \\
& =\int_{R^{2}}\left\langle\Delta \nabla \cdot\left(V V^{T}\right)+\frac{1}{\mu} \nabla \cdot(\nabla u V-V \omega(u)), \Delta w\right\rangle d x \\
& \quad+\int_{R^{2}}\left\langle\frac{1}{\mu} \nabla \cdot D(u), \Delta w\right\rangle d x \\
& \quad-\int_{R^{2}}\left\langle\Delta(u \cdot \nabla u)+\frac{1}{\mu} \nabla \cdot(u \cdot \nabla V), \Delta w\right\rangle d x \\
& \quad+\int_{R^{2}}\left\langle\Delta \nabla \cdot V^{T}, \Delta w\right\rangle d x+\int_{R^{2}} \Delta p \Delta \nabla \cdot w d x .
\end{aligned}
$$

Integrating by parts and using Lemma 4.1, the second line of (4.11) can be estimated as as follows:

$$
\begin{aligned}
& \left|\int_{R^{2}}\left\langle\Delta \nabla \cdot\left(V V^{T}\right)+\frac{1}{\mu} \nabla \cdot(\nabla u V-V \omega(u)), \Delta w\right\rangle d x\right| \\
\leq & C\|\nabla \Delta w\|\left(\|\Delta V\|\|V\|_{\infty}+\frac{1}{\mu}\|\nabla u\|\|V\|_{\infty}\right) \\
\leq & C\left(\mu+\frac{1}{\mu}\right)\|V\|_{H^{2}}\left(\|\nabla \Delta w\|^{2}+\|\nabla u\|^{2}+\frac{1}{\mu^{2}}\|\Delta V\|^{2}\right) .
\end{aligned}
$$


Next we estimate the third line of (4.11) as follows:

$$
\left|\int_{R^{2}}\left\langle\frac{1}{\mu} \nabla \cdot D(u), \Delta w\right\rangle d x\right| \leq \frac{\mu}{8}\|\nabla \Delta w\|^{2}+\frac{2}{\mu^{3}}\|\nabla u\|^{2} .
$$

Noting that $\nabla \cdot u=0$, one can estimate the fourth line of (4.11) as follows

$$
\begin{aligned}
& \quad\left|-\int_{R^{2}}\left\langle\Delta(u \cdot \nabla u)+\frac{1}{\mu} \nabla \cdot(u \cdot \nabla V), \Delta w\right\rangle d x\right| \\
& \leq\left|\int_{R^{2}}\left\langle\Delta(u \cdot \nabla u)-u \cdot \nabla \Delta u+\frac{1}{\mu} \nabla \cdot(u \cdot \nabla V)-\frac{1}{\mu} u \cdot \nabla \nabla \cdot V, \Delta w\right\rangle d x\right| \\
& \quad \quad+\left|\int_{R^{2}}\langle u \cdot \nabla \Delta w, \Delta w\rangle d x\right| \\
& =\left|\int_{R^{2}}\left\langle\Delta u \cdot \nabla u_{i}+2 \nabla_{k} u \cdot \nabla \nabla_{k} u_{i}+\frac{1}{\mu} \nabla_{j} u \cdot \nabla V_{i j}, \Delta w_{i}\right\rangle d x\right| \\
& \leq C\|\nabla \Delta w\|\left(\|\Delta u\|\|u\|_{\infty}+\|\nabla u\|\|\nabla u\|_{\infty}+\frac{1}{\mu}\|\nabla u\|\|V\|_{\infty}\right) \\
& \leq C\left(1+\frac{1}{\mu}\right)\left(\|u\|_{H^{2}}+\|V\|_{H^{2}}\right)\left(\|\nabla \Delta w\|^{2}+\|\nabla u\|_{H^{2}}^{2}\right) .
\end{aligned}
$$

To estimate the first term of the last line of (4.11), we recall the definition of $w$ in (4.9) and use (2.14) and integration by parts:

$$
\begin{aligned}
& \int_{R^{2}}\left\langle\Delta \nabla \cdot V^{T}, \Delta w\right\rangle d x \\
= & -\int_{R^{2}}\left\langle\nabla \nabla \cdot V^{T}, \nabla \Delta u+\frac{1}{\mu} \nabla \nabla \cdot V^{T}\right\rangle d x \\
\leq & -\frac{1}{\mu}\left\|\nabla \nabla \cdot V^{T}\right\|^{2}+\|\nabla \Delta u\|(\|\Delta \theta\|+\|\nabla(V \nabla \theta)\|) \\
\leq & -\frac{1}{\mu}\left\|\nabla \nabla \cdot V^{T}\right\|^{2}+8 \mu\|\nabla \Delta u\|^{2}+\frac{1}{32 \mu}\|\Delta \theta\|^{2} \\
& \quad+C \mu\|V\|_{\infty}\left(\|\nabla \Delta u\|^{2}+\frac{1}{\mu^{2}}\|\Delta \theta\|^{2}\right)+C\|\nabla V\|_{L^{4}}\|\nabla \theta\|_{L^{4}}\|\nabla \Delta u\| \\
\leq & C\left(1+\mu^{2}\right)\left(\|V\|_{H^{2}}+\frac{1}{\mu}\|\nabla \theta\|\right)\left(\|\nabla \Delta w\|^{2}+\frac{1}{\mu^{2}}\|\Delta \theta\|^{2}\right. \\
& \left.+\frac{1}{\mu^{2}}\|\Delta V\|^{2}+\|\nabla u\|_{H^{2}}^{2}\right)+8 \mu\|\nabla \Delta u\|^{2}+\frac{1}{32 \mu}\|\Delta \theta\|^{2} .
\end{aligned}
$$

Here we used the equation (2.14) of Lemma 2.2 to bring in the variable $\theta$ in order to control $\nabla \cdot V$.

It remains to estimate the last term of (4.11). To do so, we apply $\nabla \cdot$ to the momentum equation and obtain the following equation for the pressure:

$$
\Delta p=\nabla \cdot \nabla \cdot\left(V V^{T}\right)+2 \nabla \cdot \nabla \cdot V^{T}-\nabla \cdot(u \cdot \nabla u)
$$

Recalling the special structure indicated by the equation (2.14), we conclude that

$$
\nabla \cdot \nabla \cdot V^{T}=\nabla \cdot \nabla \cdot\left(J V^{T} \nabla \theta\right)
$$


Combining this with integration by parts, we can estimate the remaining term in (4.11) as follows:

$$
\begin{aligned}
& \left|\int \Delta p \Delta \nabla \cdot w d x\right|=\left|\frac{1}{\mu} \int \Delta p \nabla \cdot \nabla \cdot V^{T} d x\right| \\
= & \frac{1}{\mu}\left|\int\left[\nabla \cdot \nabla \cdot\left(V V^{T}\right)-\nabla \cdot(u \cdot \nabla u)\right] \nabla \cdot \nabla \cdot V^{T} d x\right|+\frac{2}{\mu}\left\|\nabla \cdot \nabla \cdot V^{T}\right\|^{2} \\
\leq & \frac{C}{\mu}\|V\|_{\infty}\|\Delta V\|^{2}+C\|u\|_{\infty}\left(\frac{1}{\mu^{2}}\|\Delta V\|^{2}+\|\Delta u\|^{2}\right)+C\|\nabla u\|_{\infty} \frac{1}{\mu}\|\Delta V\|\|\nabla u\| \\
& \quad+\frac{4}{\mu}\|\nabla V\|_{L^{4}}^{2}\|\nabla \theta\|_{L^{4}}^{2}+\frac{4}{\mu}\|V\|_{\infty}^{2}\|\Delta \theta\|^{2} \\
\leq & C\left(\|V\|_{H^{2}}+\|u\|_{H^{2}}+\mu^{2} \frac{1}{\mu}\|\nabla \theta\|\|V\|_{H^{2}}+\mu\|V\|_{H^{2}}^{2}\right) \\
& \quad \times\left(\|\nabla u\|_{H^{2}}^{2}+\frac{1}{\mu^{2}}\|\Delta V\|^{2}+\frac{1}{\mu^{2}}\|\Delta \theta\|^{2}\right) \\
\leq & C\left(1+\mu^{2}\right)\left(\|V\|_{H^{2}}+\|u\|_{H^{2}}+\frac{1}{\mu}\|\nabla \theta\|\right)\left(\|\nabla u\|_{H^{2}}^{2}+\frac{1}{\mu^{2}}\|\Delta V\|^{2}+\frac{1}{\mu^{2}}\|\Delta \theta\|^{2}\right) .
\end{aligned}
$$

The final step is valid provided that $\|V\|_{H^{2}} \leq 1$, a condition that can be maintained, as we will show. arguments.

Substituting (4.12)-(4.16) into (4.11), we finally arrive at

$$
\begin{aligned}
\frac{d}{d t}\|\Delta w\|^{2}+\mu\|\nabla \Delta w\|^{2} \leq & \frac{2}{\mu^{3}}\|\nabla u\|^{2}+16 \mu\|\nabla \Delta u\|^{2}+\frac{1}{32 \mu}\|\Delta \theta\|^{2} \\
& +C\left(\mu^{2}+\frac{1}{\mu}\right)\left(\|u\|_{H^{2}}+\|V\|_{H^{2}}+\frac{1}{\mu}\|\nabla \theta\|\right) \\
& \times\left(\|\nabla \Delta w\|^{2}+\|\nabla u\|_{H^{2}}^{2}+\frac{1}{\mu^{2}}\|\Delta V\|^{2}+\frac{1}{\mu^{2}}\|\Delta \theta\|^{2}\right)
\end{aligned}
$$

Since our goal is to estimate $\|\Delta V\|$, the next step is to compute the following identity:

$$
\Delta V=\nabla \nabla \cdot V+\left(\nabla^{\perp}\right)^{2} \operatorname{tr} V-\nabla^{\perp}\left[\left(\begin{array}{cc}
0 & -1 \\
1 & 0
\end{array}\right)\left(\nabla \cdot V^{T}\right)\right] .
$$

This identity is related to the Hodge decomposition for the $\Delta$ operator and can be verified by direct computation as follows:

$$
\begin{aligned}
\Delta V & =\nabla \nabla \cdot V-\nabla \times \nabla \times V \\
& =\nabla \nabla \cdot V-\left(\begin{array}{c}
\nabla_{2}\left(\nabla_{1} V_{12}-\nabla_{2} V_{11}\right)-\nabla_{1}\left(\nabla_{1} V_{12}-\nabla_{2} V_{11}\right) \\
\nabla_{2}\left(\nabla_{1} V_{22}-\nabla_{2} V_{21}\right)-\nabla_{1}\left(\nabla_{1} V_{22}-\nabla_{2} V_{21}\right)
\end{array}\right) \\
& =\nabla \nabla \cdot V+\left(\begin{array}{cc}
\nabla_{2}^{2} V_{11} & -\nabla_{1} \nabla_{2} V_{11} \\
-\nabla_{2} \nabla_{1} V_{22} & \nabla_{1}^{2} V_{22}
\end{array}\right)-\left(\begin{array}{cc}
\nabla_{2} \nabla_{1} V_{12} & -\nabla_{1}^{2} V_{12} \\
-\nabla_{2}^{2} V_{21} & \nabla_{1} \nabla_{2} V_{21}
\end{array}\right) \\
& =\nabla \nabla \cdot V+\left(\nabla^{\perp}\right)^{2} \operatorname{tr} V-\left(\begin{array}{cc}
\nabla_{2} \nabla_{1} V_{12}+\nabla_{2}^{2} V_{22} & -\nabla_{1}^{2} V_{12}-\nabla_{1} \nabla_{2} V_{22} \\
-\nabla_{2}^{2} V_{21}-\nabla_{1} \nabla_{2} V_{11} & \nabla_{1} \nabla_{2} V_{21}+\nabla_{1}^{2} V_{11}
\end{array}\right) \\
& =\nabla \nabla \cdot V+\left(\nabla^{\perp}\right)^{2} \operatorname{tr} V-\left(\begin{array}{cc}
\nabla_{2}\left(\nabla_{1} V_{12}+\nabla_{2} V_{22}\right) & -\nabla_{1}\left(\nabla_{1} V_{12}+\nabla_{2} V_{22}\right) \\
-\nabla_{2}\left(\nabla_{2} V_{21}+\nabla_{1} V_{11}\right) & \nabla_{1}\left(\nabla_{2} V_{21}+\nabla_{1} V_{11}\right)
\end{array}\right) \\
& =\nabla \nabla \cdot V+\left(\nabla^{\perp}\right)^{2} \operatorname{tr} V-\nabla^{\perp}\left[\left(\begin{array}{cc}
0 & -1 \\
1 & 0
\end{array}\right)\left(\nabla \cdot V^{T}\right)\right] .
\end{aligned}
$$


From (2.13), we notice again that

$$
\operatorname{tr} V=-\operatorname{det} V \text {. }
$$

Recalling (2.14), (4.9) and the third inequality of (4.4), we have

$$
\begin{aligned}
\|\Delta V\|^{2} \leq & C\left(\|\nabla \nabla \cdot V\|^{2}+\left\|\nabla^{\perp}\left[\left(\begin{array}{cc}
0 & -1 \\
1 & 0
\end{array}\right)\left(\nabla \cdot V^{T}\right)\right]\right\|^{2}+\left\|\left(\nabla^{\perp}\right)^{2} \operatorname{tr} V\right\|^{2}\right) \\
\leq & C \mu^{2}\left(\|\nabla \Delta u\|^{2}+\|\nabla \Delta w\|^{2}\right)+C\|V\|_{L^{\infty}}^{2}\|\Delta V\|^{2} \\
& \quad+C\|\Delta \theta\|^{2}+C\|\nabla(V \nabla \theta)\|^{2} \\
\leq & C \mu^{2}\left(\|\nabla \Delta u\|^{2}+\|\nabla \Delta w\|^{2}\right)+C\|V\|_{H^{2}}^{2}\|\Delta V\|^{2} \\
& \quad+C\|\Delta \theta\|^{2}+C\|\nabla V\|_{L^{4}}^{2}\|\nabla \theta\|_{L^{4}}^{2} \\
\leq & C \mu^{2}\left(\|\nabla \Delta u\|^{2}+\|\nabla \Delta w\|^{2}\right)+C\|\Delta \theta\|^{2} \\
& +C \mu^{2}\|\nabla V\|\|\nabla \theta\|\left(\frac{1}{\mu^{2}}\|\Delta V\|^{2}+\frac{1}{\mu^{2}}\|\Delta \theta\|^{2}\right) .
\end{aligned}
$$

Thus, if we can have the estimate

$$
\|V\|_{H^{2}}^{2}+\|\nabla \theta\|^{2} \leq \frac{1}{C}
$$

we will arrive at

$$
\|\Delta V\|^{2} \leq C\left(\mu^{2}\|\nabla \Delta u\|^{2}+\mu^{2}\|\nabla \Delta w\|^{2}+\|\Delta \theta\|^{2}\right) .
$$

It is clear now that we must estimate $\|\Delta \theta\|$. For this purpose, we will introduce another auxiliary function:

$$
\Theta=u+\frac{1}{\mu} \Delta^{-1} \nabla^{\perp} \theta
$$

We will try to find the dissipation for this new function $\Theta$. Before doing that, we combine (4.20) with (4.21) to get the estimates

$$
\|\Delta V\|^{2} \leq C \mu^{2}\left(\|\nabla \Delta u\|^{2}+\|\nabla \Delta w\|^{2}+\|\Delta \nabla \Theta\|^{2}\right)
$$

and

$$
\|\Delta \theta\|^{2} \leq 2 \mu^{2}\left(\|\nabla \Delta u\|^{2}+\|\nabla \Delta \Theta\|^{2}\right)
$$

These two inequalities (4.22) and (4.23) will let us improve (4.8) and (4.17) as:

$$
\begin{aligned}
& \frac{1}{2} \frac{d}{d t}\left(\|u\|_{H^{2}}^{2}+\|V\|_{H^{2}}^{2}\right)+\mu\|\nabla u\|_{H^{2}}^{2} \\
\leq & C(1+\mu)\left(\|u\|_{H^{2}}+\|V\|_{H^{2}}\right) \\
& \left(\|\Delta \nabla w\|^{2}+\|\nabla u\|_{H^{2}}^{2}+\|\nabla \Delta \Theta\|^{2}\right)
\end{aligned}
$$

and

$$
\begin{aligned}
& \frac{d}{d t}\|\Delta w\|^{2}+\mu\|\nabla \Delta w\|^{2} \\
& \leq \frac{2}{\mu^{3}}\|\nabla u\|^{2}+16 \mu\|\nabla \Delta u\|^{2}+\frac{\mu}{16}\left(\|\nabla \Delta \Theta\|^{2}+\|\nabla \Delta u\|^{2}\right) \\
& \quad+C\left(\mu^{2}+\frac{1}{\mu}\right)\left(\|u\|_{H^{2}}+\|V\|_{H^{2}}+\|\Delta \Theta\|\right) \\
& \quad \times\left(\|\nabla \Delta w\|^{2}+\|\nabla u\|_{H^{2}}^{2}+\|\nabla \Delta \Theta\|^{2}\right) .
\end{aligned}
$$


Our next step is to establish the energy estimates for $\|\Delta \Theta\|$. Recalling (2.14) and the definition of $\Theta$ (see equation (4.21)), we can rewrite the momentum equation as

$$
u_{t}+u \cdot \nabla u+\nabla p=\mu \Delta \Theta+\nabla \cdot\left(V V^{T}\right)+V \nabla \theta+\nabla \cdot V .
$$

Applying $\Delta$ to the above equation, multiplying (2.15) by $\frac{1}{\mu}$, and then adding the two resulting equations, we have the following equation:

$$
\begin{aligned}
& \Delta \Theta_{t}+\Delta(u \cdot \nabla u)+\frac{1}{\mu} u \cdot \nabla\left(\begin{array}{c}
-\nabla_{2} \theta \\
\nabla_{1} \theta
\end{array}\right)+\frac{1}{\mu}\left(\begin{array}{c}
-\nabla_{2} u \cdot \nabla \theta \\
\nabla_{1} u \cdot \nabla \theta
\end{array}\right)+\Delta \nabla p \\
= & \mu \Delta^{2} \Theta+\Delta \nabla \cdot\left(V V^{T}\right)+\Delta(V \nabla \theta)+\Delta \nabla \cdot V+\frac{1}{2 \mu}\left(\begin{array}{c}
\Delta u_{1} \\
\Delta u_{2}
\end{array}\right) .
\end{aligned}
$$

Taking the $L^{2}$ inner product of the above equation (4.26) with $\Delta \Theta$, we can get

$$
\begin{aligned}
& \frac{1}{2} \frac{d}{d t} \int|\Delta \Theta|^{2} d x+\mu\|\nabla \Delta \Theta\|^{2} \\
& +\int\langle u \cdot \nabla \Delta \Theta, \Delta \Theta\rangle+\langle\nabla \Delta p, \Delta \Theta\rangle d x \\
=- & \int\langle\Delta(u \cdot \nabla u)-u \cdot \nabla \Delta u, \Delta \Theta\rangle d x \\
& -\int\left\langle\frac{1}{\mu}\left(\begin{array}{c}
-\nabla_{2} u \cdot \nabla \theta \\
\nabla_{1} u \cdot \nabla \theta
\end{array}\right), \Delta \Theta\right\rangle d x \\
& +\int\left\langle\Delta \nabla \cdot\left(V V^{T}\right)+\Delta(V \nabla \theta), \Delta \Theta\right\rangle d x \\
& +\int\left\langle\frac{1}{2 \mu}\left(\begin{array}{c}
\Delta u_{1} \\
\Delta u_{2}
\end{array}\right), \Delta \Theta\right\rangle d x \\
& +\int\langle\Delta \nabla \cdot V, \Delta \Theta\rangle d x .
\end{aligned}
$$

We estimate the terms in the above estimate one by one. First, by using integration by parts, it is easy to get

$$
\int\langle u \cdot \nabla \Delta \Theta, \Delta \Theta\rangle+\langle\nabla \Delta p, \Delta \Theta\rangle d x=0 .
$$

By Lemma 4.1, the third and fourth lines of the equation (4.27) can be estimated as follows

$$
\begin{aligned}
& \left|-\int\langle\Delta(u \cdot \nabla u)-u \cdot \nabla \Delta u, \Delta \Theta\rangle d x\right| \\
& \quad+\left|-\int\left\langle\frac{1}{\mu}\left(\begin{array}{c}
-\nabla_{2} u \cdot \nabla \theta \\
\nabla_{1} u \cdot \nabla \theta
\end{array}\right), \Delta \Theta\right\rangle d x\right| \\
& \leq C\left(\|u\|_{\infty}\|\Delta u\|+\|\nabla u\|_{\infty}\|\nabla u\|\right)\|\Delta \nabla \Theta\|+\frac{1}{\mu}\|\nabla u\|\|\nabla \theta\|_{L^{4}}\|\Delta \Theta\|_{L^{4}} \\
& \leq C\|u\|_{H^{2}}\left(\|\nabla u\|_{H^{2}}^{2}+\|\Delta \nabla \Theta\|^{2}\right)+\frac{1}{\mu}\|\nabla u\|\|\nabla \theta\|^{\frac{1}{2}}\|\Delta \Theta\|^{\frac{1}{2}}\|\Delta \theta\|^{\frac{1}{2}}\|\Delta \nabla \Theta\|^{\frac{1}{2}} \\
& \leq C\left(\|u\|_{H^{2}}+\|\Delta \Theta\|\right)\left(\|\nabla u\|_{H^{2}}^{2}+\|\Delta \nabla \Theta\|^{2}\right) .
\end{aligned}
$$


Next, by using Lemma 4.1, the fifth line in (4.27) can be estimated as follows

$$
\begin{aligned}
& \quad\left|\int\left\langle\Delta \nabla \cdot\left(V V^{T}\right)+\Delta(V \nabla \theta), \Delta \Theta\right\rangle d x\right| \\
& \leq C \mu\|V\|_{\infty}\left(\frac{1}{\mu^{2}}\|\Delta V\|^{2}+\|\nabla \Delta \Theta\|^{2}+\frac{1}{\mu^{2}}\|\Delta \theta\|^{2}\right) \\
& \quad+C\|\nabla V\|_{L^{4}}\|\nabla \theta\|_{L^{4}}\|\nabla \Delta \Theta\| \\
& \leq C(1+\mu)\left(\|u\|_{H^{2}}+\|V\|_{H^{2}}+\|\Delta \Theta\|\right)\left(\|\nabla u\|_{H^{2}}^{2}+\|\nabla \Delta \Theta\|^{2}+\|\nabla \Delta w\|^{2}\right) .
\end{aligned}
$$

In the estimates, we have used the definition of $\Theta$ (see (4.21)), and the estimates (4.22) and (4.23). It is easy to estimate the sixth line in (4.27):

$$
\left|\int\left\langle\frac{1}{2 \mu}\left(\begin{array}{c}
\Delta u_{1} \\
\Delta u_{2}
\end{array}\right), \Delta \Theta\right\rangle d x\right| \leq \frac{\mu}{8}\|\nabla \Delta \Theta\|^{2}+\frac{1}{2 \mu^{3}}\|\nabla u\|^{2}
$$

Now by the definition of $w$ (see (4.9)), we can estimate the last term of (4.27).

$$
\begin{aligned}
\left|\int\langle\Delta \nabla \cdot V, \Delta \Theta\rangle d x\right| & =\left|\int\langle\nabla \nabla \cdot V, \nabla \Delta \Theta\rangle d x\right| \\
& \leq \frac{\mu}{8}\|\nabla \Delta \Theta\|^{2}+\frac{2}{\mu}\|\nabla \nabla \cdot V\|^{2} \\
& \leq \frac{\mu}{8}\|\nabla \Delta \Theta\|^{2}+4 \mu\left(\|\nabla \Delta u\|^{2}+\|\nabla \Delta w\|^{2}\right) .
\end{aligned}
$$

Combining all these estimates (4.27)-(4.32), we arrive at

$$
\begin{aligned}
& \quad \frac{d}{d t}\|\Delta \Theta\|^{2}+\mu\|\nabla \Delta \Theta\|^{2} \\
& \leq \frac{C}{\mu^{3}}\|\nabla u\|^{2}+C \mu\|\nabla \Delta u\|^{2}+4 \mu\|\nabla \Delta w\|^{2} \\
& \quad+C(1+\mu)\left(\|u\|_{H^{2}}+\|V\|_{H^{2}}+\|\Delta \Theta\|\right) \\
& \quad \times\left(\|\nabla u\|_{H^{2}}^{2}+\|\nabla \Delta \Theta\|^{2}+\|\nabla \Delta w\|^{2}\right) .
\end{aligned}
$$

Multiplying (4.25) by an appropriately big constant, say 8 , and then adding the resulting inequality with (4.33), we arrive at

$$
\begin{aligned}
& \frac{d}{d t}\left(8\|\Delta w\|^{2}+\|\Delta \Theta\|^{2}\right)+\frac{\mu}{2}\left(\|\nabla \Delta w\|^{2}+\|\nabla \Delta \Theta\|^{2}\right) \\
\leq & C\left(\mu^{2}+\frac{1}{\mu}\right)\left(\|u\|_{H^{2}}+\|V\|_{H^{2}}+\|\Delta \Theta\|\right) \\
& \times\left(\|\nabla \Delta w\|^{2}+\|\nabla u\|_{H^{2}}^{2}+\|\nabla \Delta \Theta\|^{2}\right) \\
& +\frac{C}{\mu^{3}}\|\nabla u\|^{2}+C \mu\|\nabla \Delta u\|^{2} .
\end{aligned}
$$

Multiplying (4.24) by $2 C$, and adding the resulting inequality with (4.34), we obtain that 


$$
\begin{aligned}
& \frac{d}{d t}\left[C\left(\|u\|_{H^{2}}^{2}+\|V\|_{H^{2}}^{2}\right)+\|\Delta \Theta\|^{2}+8\|\Delta w\|^{2}\right] \\
& \quad+\frac{\mu}{2}\left(\|\nabla u\|_{H^{2}}^{2}+\|\nabla \Delta w\|^{2}+\|\nabla \Delta \Theta\|^{2}\right) \\
& \leq C\left(\mu^{2}+\frac{1}{\mu}\right)\left(\|u\|_{H^{2}}+\|V\|_{H^{2}}+\|\Delta \Theta\|\right) \\
& \quad \times\left(\|\nabla \Delta w\|^{2}+\|\nabla u\|_{H^{2}}^{2}+\|\nabla \Delta \Theta\|^{2}\right)+\frac{C}{\mu^{3}}\|\nabla u\|^{2} .
\end{aligned}
$$

Now we impose the smallness assumption on the initial data

$$
\left\|u_{0}\right\|_{H^{2}}^{2}+\left\|V_{0}\right\|_{H^{2}}^{2}+\left\|\nabla \theta_{0}\right\|^{2} \leq \frac{\mu^{6}}{M\left(1+\mu^{8}\right)},
$$

where $M>0$ is sufficiently large. We point out here that we have no smallness assumption on $\theta_{0}$ itself. By (4.36) and the definition of $\Theta$, it is easy to see that

$$
\begin{aligned}
& \left\|u_{0}\right\|_{H^{2}}+\left\|V_{0}\right\|_{H^{2}}+\left\|\Delta \Theta_{0}\right\| \\
\leq & 2\left(1+\frac{1}{\mu}\right)\left(\left\|u_{0}\right\|_{H^{2}}+\left\|V_{0}\right\|_{H^{2}}+\left\|\nabla \theta_{0}\right\|\right) \\
\leq & 4\left(1+\frac{1}{\mu}\right) \sqrt{\frac{\mu^{6}}{M\left(1+\mu^{8}\right)}} \leq \frac{1}{4} \frac{\mu^{2}}{4 C\left(\mu^{3}+1\right)} .
\end{aligned}
$$

Note that the above computation is valid independent of the magnitude of the viscosity $\mu$ provided that $M>C^{3}$. By continuity, there exists a positive time $T^{*}$ such that

$$
\|u\|_{H^{2}}+\|V\|_{H^{2}}+\|\Delta \Theta\| \leq \frac{\mu^{2}}{4 C\left(\mu^{3}+1\right)} .
$$

for all $0 \leq t<T^{*}$.

Now obviously (4.19) is true for $0 \leq t<T^{*}$ due to (4.37). Then (4.35) is valid for $0 \leq t<T^{*}$. Using (4.37), we improve (4.35) as

$$
\begin{aligned}
& \frac{d}{d t}\left[C\left(\|u\|_{H^{2}}^{2}+\|V\|_{H^{2}}^{2}\right)+\|\Delta \Theta\|^{2}+8\|\Delta w\|^{2}\right] \\
& \quad+\frac{\mu}{4}\left(\|\nabla u\|_{H^{2}}^{2}+\|\nabla \Delta w\|^{2}+\|\nabla \Delta \Theta\|^{2}\right) \leq \frac{C}{\mu^{3}}\|\nabla u\|^{2} .
\end{aligned}
$$

Consequently, using the definition of $\Theta$ and $w$, and integrating the above equation with respect to $t$ gives

$$
\begin{aligned}
& \|u\|_{H^{2}}^{2}+\|V\|_{H^{2}}^{2}+\|\Delta \Theta\|^{2}+\|\Delta w\|^{2} \\
& \quad+\frac{\mu}{4} \int_{0}^{T^{*}}\left(\|\nabla \Delta w\|^{2}+\|\nabla u\|_{H^{2}}^{2}+\|\nabla \Delta \Theta\|^{2}\right) d t \\
& \leq C\|u\|_{H^{2}}^{2}+C\|V\|_{H^{2}}^{2}+\|\Delta \Theta\|^{2}+8\|\Delta w\|^{2} \\
& \quad+\frac{\mu}{4} \int_{0}^{T^{*}}\left(\|\nabla \Delta w\|^{2}+\|\nabla u\|_{H^{2}}^{2}+\|\nabla \Delta \Theta\|^{2}\right) d t \\
& \leq C\left(\left\|u_{0}\right\|_{H^{2}}^{2}+\left\|V_{0}\right\|_{H^{2}}^{2}\right)+\left\|\Delta \Theta_{0}\right\|^{2}+8\left\|\Delta w_{0}\right\|^{2}+\frac{C}{\mu^{3}} \int_{0}^{\infty}\|\nabla u\|^{2} d t \\
& \leq C\left(1+\frac{1}{\mu^{2}}\right)\left(\left\|u_{0}\right\|_{H^{2}}^{2}+\left\|V_{0}\right\|_{H^{2}}^{2}+\left\|\nabla \theta_{0}\right\|^{2}\right)+\frac{C}{\mu^{4}}\left(\left\|u_{0}\right\|_{L^{2}}^{2}+\left\|V_{0}\right\|_{H^{2}}^{2}\right),
\end{aligned}
$$


where in the last inequality, we used the energy law (4.1) and $\operatorname{tr} V=-\operatorname{det} V$. Recalling our smallness assumption on the initial data (4.36), we have

$$
\begin{aligned}
& \left(\|u\|_{H^{2}}+\|V\|_{H^{2}}+\|\Delta \Theta\|\right)^{2} \\
\leq & 2\left(\|u\|_{H^{2}}^{2}+\|V\|_{H^{2}}^{2}+\|\Delta \Theta\|^{2}+\|\Delta w\|^{2}\right. \\
& \left.+\frac{\mu}{4} \int_{0}^{T^{*}}\left(\|\nabla \Delta w\|^{2}+\|\nabla u\|_{H^{2}}^{2}+\|\nabla \Delta \Theta\|^{2}\right) d t\right) \\
\leq & \frac{C\left(\mu^{2}+\mu^{6}\right)}{M\left(1+\mu^{8}\right)} \leq \frac{1}{2}\left[\frac{\mu^{2}}{4 C\left(\mu^{3}+1\right)}\right]^{2} .
\end{aligned}
$$

Estimate (4.39) implies that (4.37) remains true for all the latter time with a uniform constant $C$ independent of $\mu$ and $t$.

In conclusion, we have the following theorem, which states that if the initial state has small strain $V$ (and $\nabla \theta$ ), then the viscoelastic system does posses a global classical solution.

THEOREM 4.2. Let $\Omega \in R^{2}$ be either a bounded domain with smooth boundary or the entire space $R^{2}$. Suppose that $u_{0} \in H^{k}(\Omega), V_{0} \in H^{k}(\Omega)$ and $\nabla \theta_{0} \in H^{k-1}(\Omega)$, for some integer $k \geq 2$. Suppose further that assumptions (2.12) on the initial data are satisfied. Then there exists a unique global classical solution for the Cauchy problem (2.10)-(2.11) or the initial boundary value problem (2.10)-(2.11) such that

$$
\|u\|_{H^{2}}^{2}+\|V\|_{H^{2}}^{2}+\mu \int_{0}^{\infty}\left(\|\nabla \Delta w\|^{2}+\|\nabla u\|_{H^{2}}^{2}\right) d t \leq \frac{\mu^{4}}{C^{2}\left(1+\mu^{3}\right)^{2}},
$$

provided the initial data satisfies

$$
\left\|u_{0}\right\|_{H^{2}}^{2}+\left\|V_{0}\right\|_{H^{2}}^{2}+\left\|\nabla \theta_{0}\right\|_{H^{1}}^{2} \leq \frac{\mu^{6}}{M\left(1+\mu^{8}\right)}
$$

for some sufficiently large constant $M\left(M>C^{3}\right)$ independent of $\mu$ and $t$.

We will finish the paper by making several remarks concerning the results of the theorem.

REMARK 4.3. It is very interesting to study whether in the limit as the viscosity $\mu$ tends to zero, the solutions of system (1.12) or (2.10) converge to those of the following inviscid system:

$$
\left\{\begin{aligned}
u_{t}+u \cdot \nabla u+\nabla p & =\nabla \cdot\left(F F^{T}\right) \\
F_{t}+u \cdot \nabla F & =\nabla u F \\
\nabla \cdot u & =0
\end{aligned}\right.
$$

Unfortunately, our theorem cannot give any information for that since the bound in (4.40) tends to zero as $\mu$ tends to zero.

REMARK 4.4. The above theorem only gives results for small strain. It cannot be generalized to the large viscosity cases, e.g., the Navier-Stokes equations.

REMARK 4.5. The results in Theorem 4.1 are still true for a general isotropic elastic energy function $W(F)$ if the usual Legendre-Hadamard ellipticity condition is imposed upon the linearized elasticity tensor. For this part of the argument, we refer to our recent work in [30]. 
REMARK 4.6. In the case of $\Omega=\mathrm{T}^{2}$, the two dimensional torus, we can also derive the small data global existence results from the results in [30]. To see this, we set

$$
\bar{\theta}_{0}=\frac{1}{\left|\mathrm{~T}^{2}\right|} \int_{\mathrm{T}^{2}} \theta_{0} d x
$$

By Poincaré's inequality, we have

$$
\left\|\theta_{0}-\bar{\theta}_{0}\right\|_{L^{\infty}} \leq C\left\|\nabla \theta_{0}\right\|_{H^{1}}
$$

Thus, if we define

$$
Q_{0}=\left(\begin{array}{cc}
\cos \bar{\theta}_{0} & -\sin \bar{\theta}_{0} \\
\sin \bar{\theta}_{0} & \cos \bar{\theta}_{0}
\end{array}\right)
$$

then

$$
\left.(I+V) Q\right|_{t=0}=Q_{0}+E_{0}
$$

where $E_{0}$ is a small disturbance. In [30], we considered the general viscoelastic models and proved global existence near constant equilibrium. However, our proof here still provides a different and perhaps better understanding of the physical background.

REMARK 4.7. By defining the rotation conjugate of $V$ as

$$
V_{1}=Q^{T} V Q
$$

we see that $F=Q\left(I+V_{1}\right)$, which is the decomposition in [34]. However, the analysis will be exactly the same in this case.

5. Acknowledgements The authors want to thank Professors Weinan E, Fanghua Lin and Noel Walkington for many helpful discussions.

\section{REFERENCES}

[1] R. Agemi, Global existence of nonlinear elastic waves, Invent. Math. 142, 2, 225-250, 2000.

[2] S. Alinhac, Blowup for Nonlinear Hyperbolic Equations, Birkhäuser, 1995.

[3] S. Alinhac, The null condition for quasilinear wave equations in two space dimensions. I, Invent. Math., 145(3), 597-618, 2001.

[4] S. Alinhac, The null condition for quasilinear wave equations in two space dimensions. II, Amer. J. Math., 123(6), 1071-1101, 2001.

[5] T.J. Burns, Similarity and bifurcation in unstable viscoplastic shear, SIAM J. Appl. Math., 49, (1), 314-329, 1989.

[6] J.Y. Chemin and N. Masmoudi, About lifespan of regular solutions of equations related to viscoelastic fluids, SIAM J. Math. Anal., 33(1), 84-112, 2001.

[7] D. Christodoulou, Global existence of nonlinear hyperbolic equations for small data, Comm. Pure. Appl. Math., 39, 267-286, 1986.

[8] M. Doi and S.F. Edwards, The Theory of Polymer Dynamics, Oxford Science Publication, 1986.

[9] D. Ebin, Global solutions of the equations of elastodynamics of incompressible neo-Hookean materials, Proc. Nat. Acad. Sci. U.S.A., 90, 3802-3805, 1993.

[10] D. Ebin, Global solutions of the equations of elastodynamics of incompressible materials, Electron. Res. Announc. Amer. Math. Soc., 2, 50-59, 1996.

[11] J. Ericksen, Conservation laws for liquid crystals, Trans. Soc. Rheol., 5, 22-34, 1961.

[12] E. Fernández-Cara, F. Guillén and R.R. Ortega, Some theoretical results for viscoplastic and dilatant fluids with variable density, Nonlinear Anal., 28(6), 1079-1100, 1997.

[13] C. Foias, Navier-Stokes Equations, Texas A \& M Lecture Notes, 2002. 
[14] J.M. Greenberg and A. Nouri, Antiplane shearing motions of a viscoplastic solid, SIAM J. Math. Anal., 24, 4, 943-967, 1993.

[15] M.E. Gurtin, An Introduction to Continuum Mechanics. Mathematics in Science and Engineering, Academic Press, New York, London, 158, 1981.

[16] L. Hörmander, The lifespan of classical solutions of non-linear equations, Mittag-Leffler Institute reports, 5, 1985.

[17] J. Hron, J. Málek, J. Nečas and K.R. Rajagopal, Numerical simulations and global existence of solutions of two-dimensional flows of fluids with pressure- and shear-dependent viscosities, Math. Comut. Simulation, 61(3-6), 297-315, 2003.

[18] F. John, Rotation and strain, Comm. Pure. Appl. Math., 14, 391-413, 1961.

[19] F. John, Formation of singularities in one-dimensional nonlinear wave propagation, Comm. Pure. Appl. Math., 27, 377-405, 1974.

[20] D.D. Joseph, Instability of the rest state of fluids of arbitrary grade greater than one, Arch. Rational Mech. Anal., 75(3), 251-256, 1980/81.

[21] S. Klainerman, Uniform decay estimates and the Lorentz invariance of the classical wave equation, Comm. Pure Appl. Math., 38, 321-332, 1985.

[22] S. Klainerman, The null condition and global existence to nonlinear wave equations. Nonlinear Systems of Partial Differential Equations in Applied Mathematics, Santa Fe, N. M., 1, 293-326, 1984. Lectures in Appl. Math. 23 Amer. Math. Soc., Providence, RI, 1986.

[23] S. Klainerman and A. Majda, Singular limits of quasilinear hyperbolic system with large parameters and the incompressible limit of compressible fluids, Comm. Pure Appl. Math., 34, 481-524, 1981.

[24] S. Klainerman and T.C. Sideris, On almost global existence for nonrelativistic wave equations in 3D, Comm. Pure Appl. Math., 49, 307-322, 1996.

[25] S.N. Kruzkov, First order quasilinear equations in several independent variables, Math. USSR Sbornik, 10(2), 217-243, 1970.

[26] O.A. Ladyzhenskaya and G.A. Seregin, On the regularity of solutions of two-dimensional equations of the dynamics of fluids with nonlinear viscosity, Zapiski Nauchn Seminar. POMI., 259, 145-166, 1999.

[27] R.G. Larson, The structure and rheology of complex fluids, Oxford, 1995.

[28] Z. Lei, Global existence of classical solutions for some Oldroyd-B model via the incompressible limit, Chin. Ann. Math. Ser.B, 27(5), 565-580, 2006.

[29] Z. Lei and Y. Zhou, Global existence of classical solutions for 2D Oldroyd model via the incompressible limit, SIAM J. Math. Anal., 37(3), 797-814, 2005.

[30] Z. Lei, C. Liu and Y. Zhou, Global solutions for incompressible viscoelastic fluids, Arch. Ration. Mech. Anal., to appear, 2007.

[31] T.T. Li and Y.M. Chen, Global classical solutions for nonlinear evolution equations, Pitman Monographs and Surveys in Pure and Appl. Math., 45. Longman Scientific and Technical, Harlow; copublished in the United States with John Wiley and Sons, Inc., New York, 1992.

[32] F.H. Lin, C. Liu and P. Zhang, On hydrodynamics of viscoelastic fluids, Comm. Pure Appl. Math., 58(11), 1437-1471, 2005.

[33] P.L. Lions and N. Masmoudi, Global solutions for some Oldroyd models of non-Newtonian flows, Chinese Ann. Math. Ser. B, 21(2), 131-146, 2000.

[34] C. Liu and N.J. Walkington, An Eulerian description of fluids containing visco-hyperelastic particles, Arch. Rat. Mech Ana., 159, 229-252, 2001.

[35] J. Málek, J. Nečas and K.R. Rajagopal, Global analysis of solutions of the flows of fluids with pressure-dependent viscosities, Arch. Ration. Mech. Anal., 3(165), 243-269, 2002.

[36] C. Peskin, A random-walk interpretation of the incompressible Navier-Stokes equations, Comm. Pure Appl. Math., 38, 845-852, 1985.

[37] W.R. Schowalter, Mechanics of Non-Newtonian Fluids, Pergamon Press, 1978.

[38] T.C. Sideris, Nonresonance and global existence of prestressed nonlinear elastic waves, Ann. of Math., 151, 849-874, 2000.

[39] T.C. Sideris and B. Thomases, Global existence for 3D incompressible isotropic elastodynamics via the incompressible limit, Comm. Pure Appl. Math., 57, 1-39, 2004.

[40] M. Slemrod, Constitutive relations for Rivlin-Erichsen fluids based on generalized rational approximation, Arch. Ration. Mech. Anal., 1, 146, 73-93, 1999.

[41] L. Tartar, Compensated compactness and applications to partial differential equations, R. K. Knops, editor, Research Notes in Mathematics, Nonlinear Analysis and Mechanics, Neriot Watt Symposium, Pitman Press, volume 4, 1979.

[42] R. Teman, Navier-Stokes Equations, North Holland, Amsterdam, 1977.

[43] A. Tordesillas and J. Shi, Indentation of a double shearing dilatant granular material by a smooth rigid wedge, Quart. J. Mech. Appl. Math., 51(4), 633-646, 1998. 